\title{
MINERALIZAÇÃO DE PALHA DE MILHO E ADSORÇÃO DE CARBONO, CÁLCIO, MAGNÉSIO E POTÁSSIO EM SUBSTRATOS COM CAULINITA NATURAL E GOETHITA SINTÉTICA ${ }^{(1)}$
}

\author{
L. S. SILVA ${ }^{(2)} \&$ H. BOHNEN ${ }^{(3)}$
}

\begin{abstract}
RESUMO
Carbono e nutrientes liberados na decomposição de resíduos orgânicos podem sofrer vários tipos de interação com diferentes componentes da fase mineral, alterando sua dinâmica no solo. Com o objetivo de avaliar a retenção de carbono, $\mathrm{Ca}^{2+}, \mathrm{Mg}^{2+} \mathrm{eK}^{+}$em caulinita natural e goethita sintética, foi realizado um experimento com mistura de palha de milho em substratos inorgânicos preparados com areia e quantidades conhecidas de cada componente. Nesses substratos, foram realizadas percolações com água destilada aos 7, 15, 33,68 e 113 dias de incubação, em temperatura ambiente, sendo as massas dos elementos liberados acumuladas no tempo. Após duzentos dias de incubação, foi determinado o carbono orgânico total que permaneceu nos substratos. A soma das massas de $\mathrm{Ca}^{2+}, \mathrm{Mg}^{2+}, \mathrm{K}^{+}$e carbono, liberados com a água de percolação, foi inversamente relacionada com a massa dos componentes inorgânicos nos substratos, exceto para o $\mathrm{K}^{+}$nos substratos com goethita. A caulinita e a goethita apresentaram diferente retenção do $\mathrm{Ca}^{2+}, \mathrm{Mg}^{2+} \mathrm{e} \mathrm{K}^{+} \mathrm{liberados}$, independentemente da área superficial, o que pode estar relacionado com suas distintas características eletroquímicas superficiais. A goethita foi mais efetiva do que a caulinita em reter carbono da solução do solo e acumular carbono orgânico total, sendo sua maior reatividade diretamente relacionada com sua superfície específica.
\end{abstract}

Termos de indexação: incubação, percolação, decomposi ção, minerais.

(1) Trabal ho apresentado como resumo no III EBSH (24 a 26/11/1999). Santa Maria (RS). Recebido para publicação em abril de 2000 e aprovado em janeiro de 2001.

(2) Doutorando em Ciência do Solo no Departamento de Solos da Faculdade de Agronomia, Universidade Federal do Rio Grande do Sul - UFRGS, Caixa Postal 776, CEP 90.001-970 Porto Alegre (RS). E-mail: Issilva@vortex.ufrgs.br

(3) Engenheiro-Agrônomo, Ph.D. Colaborador convidado. Departamento deSolos, FaculdadedeAgronomia, UFRGS, Caixa Postal 776, CEP 90.001-970, Porto Alegre (RS). E-mail: bohnen@conex.com.br 


\title{
SUMMARY: CORN STRAW MINERALIZATION AND CARBON, CALCIUM, MAGNESIUM AND POTASSIUM ADSORPTION IN MIXTURES WITH KAOLINITE AND SYNTHETIC GOETHITE
}

\begin{abstract}
Carbon and nutrients released from themineral ization of organic materials can interact with mineral components changing their dynamics in the soil. In this study, mixtures of corn straw, kaolinite, synthetic goethite, and quartz sand were leached with water after 7 , $15,33,68$ and 113 days of incubation at room temperature. Concentrations of solublecarbon, calcium, magnesium, and potassium were measured and accumulated over time. After 200 days, the total carbon remaining in the mixture was quantified. Total calcium, magnesium, and potassium percol ated during theexperiment wereinversely corredated with the amount of kaolinite and goethitein the mixtures, except for potassium in the treatment with goethite. Adsorption of calcium, magnesium, and potassium did not relatewith specific surfacearea of kaol initeand goethite Otherwise, goethitewas more efficient than kaol inite in retaining carbon and this property was clearly related with its surface area.
\end{abstract}

Index terms: incubation, percolation, decomposition, minerals.

\section{NTRODUÇÃO}

A decomposição dos resíduos de origem vegetal tem papel fundamental na dinâmica da matéria orgânica, nas características químicas, físicas e biológicas do solo e grande importância no fenômeno de aquecimento global (Stevenson, 1994). Durante os processos de decomposição e mineralização dos resíduos de culturas, compostos orgânicos e nutrientes sãoliberados para a solução do solo, onde podem sofrer vários tipos de interação com a fase mineral (Cornejo \& Hermosín, 1996).

Dessa forma, íons ou moléculas da solução podem ser adsorvidos nas superfícies de argilominerais ou óxidos, modificando o seu comportamento no solo, principalmente quanto à disponibilidade para as plantas e transporte no perfil (Vasudevan \& Stone, 1998).

Considerando essa interação, as moléculas orgânicas podem tornar-se protegidas fisicamente, dificultando o uso do carbono orgânico como um substrato pel os microrganismos (Zech et al., 1997). Este fenômeno pode, portanto, alterar a taxa de decomposição dos materiais orgânicos adicionados e, conseqüentemente, influir na dinâmica e na estabilização da matéria orgânica no solo.

Segundo diversos autores (Greenland, 1971; Oades et al., 1989; Sposito, 1989; Cornejo\& Hermosín 1996), a interação entre íons e moléculas com os minerais presentes no solo é devida a diferentes mecanismos (forças de van der Waals, atração eletrostática, protonação, pontes de $\mathrm{H}$, ponte de água e cátions, troca de ligantes, entre outros), dependendo da natureza e de propriedades intrínsecas das espécies envolvidas, especial mente quanto às características de carga e superfície, da quantidade de água no sistema e do $\mathrm{pH}$ do meio. A estabilidade destes mecanismos depende do tipo de ligação existente, dos átomos envol vidos e da afinidade com os grupos aos quais estãoligados, podendo dois ou mais mecanismos ocorrer simultaneamente no solo (M ortland, 1986).

U ma vez que as reações de superfície que influenciam a dinâmica dos compostos orgânicos e dos nutrientes liberados para a solução do solo dependem das características e da quantidade dos componentes inorgânicos (Martin \& Haider, 1986), uma alternativa para se estudar a influência de minerais, presentes simultaneamente eem variada quantidade nos solos, seria a adição de resíduos de culturas em substratos inorgânicos, preparados com quantidades conhecidas de cada um e avaliados durante determinado período de incubação.

Nesses casos, apesar das limitações para se comparar com o ambiente do solo, exclui-se a interferência dos componentes orgânicos naturalmente existentes na matriz mineral. Dessa forma, a massa de carbono e de nutrientes liberada com a água de percolação poderia servir como um índice para avaliar a dinâmica que envolve a mineralização dos resíduos e a interação dos componentes presentes no sistema.

O objetivo destetrabal ho foi avaliar a capacidade de diferentes substratos inorgânicos, preparados pela mistura de determinadas proporções de areia com caulinita natural ou goethita sintética, em reter carbono e nutrientes liberados durante a decomposição de palha de milho.

\section{MATERIAL E MÉTODOS}

\section{Preparo dos substratos inorgânicos}

Foram preparados substratos inorgânicos, misturando diferentes proporções de: (a) areia (lavada 
inicialmente com uma mistura sulfocrômica de $\mathrm{K}_{2} \mathrm{Cr}_{2} \mathrm{O}_{7} 1,25 \mathrm{~mol} \mathrm{~L}^{-1} \mathrm{com} 176 \mathrm{~mL}$ de $\mathrm{H}_{2} \mathrm{SO}_{4}$ concentrado para retirada de material orgânico e, em seguida, com água destilada até redução da condutividade elétrica para valor constante $14 \mu \mathrm{S} \mathrm{cm}^{-1}$ - e, posteriormente, seca em estufa a $60^{\circ} \mathrm{C}$ ); (b) caulinita natural (caulin comercial marca Raabe de Pantano Grande (RS), selecionado pela presença de caulinita na análise de DRX, fração menor que $105 \mu \mathrm{m}$, quefoi lavado com água destilada e seco em estufa a $60^{\circ} \mathrm{C}$ e, posteriormente, moído) ou; (c) óxido de ferro sintético (preparadoa partir de uma solução de $\mathrm{FeCl}_{3} 0,1 \mathrm{~mol} \mathrm{~L}^{-1} \mathrm{com} \mathrm{pH}$ ajustado para $6,5 \mathrm{com} \mathrm{NaHCO}_{3} 0,1 \mathrm{~mol} \mathrm{~L}^{-1}$. Após formação do precipitado, o material foi lavado com água destilada, seco em estufa a $50^{\circ} \mathrm{C}$ e, posteriormente, moído e caracterizado como goethita pel o padrão de DRX).

Para cada componente inorgânico utilizado, foi determinada a superfície específica (SE), após secagem da amostra com $\mathrm{P}_{2} \mathrm{O}_{5}$ e umedecimento com acetato de $\mathrm{K}$ saturado com água, considerando a ocorrência de adsorção monocamada de moléculas de $\mathrm{H}_{2} \mathrm{O}$ na superfície das partículas com o equivalente de uma molécula igual a $0,108 \mathrm{~nm}^{2}$ (Quirk, 1955). Foram então estabelecidos os tratamentos (Quadro 1).

\section{Preparo dos tubos de decomposição}

Utilizaram-se, como resíduo orgânico, fol has de plantas de milho (Zea mays L.), coletadas 120 dias após a semeadura, secas em estufa e moídas $(<1 \mathrm{~mm}$ ), que apresentaram teores de $C$ (método Walkley-Black) N, P, K, Ca e Mg de 419; 16; 1,8; 11; 2,2 e 3,2 $\mathrm{g} \mathrm{kg}^{-1}$, respectivamente, após digestão com $\mathrm{H}_{2} \mathrm{SO}_{4}$ e $\mathrm{H}_{2} \mathrm{O}_{2}$, conforme descrito em Tedesco et al. (1995). Foi separada a fração maior que 0,1 mm para ser misturada aos substratos (Quadro 1), na quantidade equivalente a $5 \mathrm{~g} \mathrm{~kg}^{-1}$.
As amostras de cada tratamento (aproximadamente $200 \mathrm{~g}$ ), homogeneamente misturadas com a pal ha, foram acondicionadas, em duplicata, em tubos dePVC de $5 \mathrm{~cm}$ dediâmetroe $10 \mathrm{~cm}$ dealtura, tendo, na parte inferior, um tampão perfurado com uma tela de nylon e uma camada de areia lavada (25 g). O substrato de cada tubo foi umedecido, a partir da superfície, comágua destilada mais $2 \mathrm{~mL}$ de solução de um solo aluvial extraída por centrifugação para servir de fonte de inóculo à colonização de microrganismos, suficiente para atingir $10,0 \%$ de umidade gravimétrica. Durante o período de avaliação, os tubos foram mantidos no laboratório em temperatura ambiente e a umi dade reposta com água destilada a cada 10-15 dias.

\section{Avaliações}

Aos 7, 15, 33, 68 e 113 dias de decomposição da pal ha, foram realizadas percolações pela adição de $20 \mathrm{~mL}$ de água destilada e aplicada uma sucção $(0,7 \mathrm{~atm})$ na parteinferior dos tubos, com uso de uma bomba de vácuo conectada a um frasco kitassato que continha, na parte superior, um funil revestido de silicone para encaixar o tubo de PVC, até recolher aproximadamente $20 \mathrm{~mL}$ de percolado. Após centrifugação do percolado (2.500 rpm por 5 min.), foi retirada uma alíquota para análise de carbono orgânico solúvel por colorimetria $(580 \mathrm{~nm})$, com digestão da amostra por $4 \mathrm{~h} \mathrm{a} 60^{\circ} \mathrm{C}$ com uma mistura sulfocrômica de $\mathrm{K}_{2} \mathrm{Cr}_{2} \mathrm{O}_{7} 1,25 \mathrm{~mol} \mathrm{~L}^{-1} \mathrm{com} 80 \mathrm{~mL}$ de $\mathrm{H}_{2} \mathrm{SO}_{4}$ concentrado, na relação amostra : mistura de 1 : 1 , sendo usado um fator de 1,35 em relação à curva de glicose, procedimento correlacionado ( $r=0,997)$ com o método descrito em Moore (1985).

$\mathrm{O}$ restante do percolado foi passado em filtro 0,2 $\mathrm{mm}$ versapor (Gelman Sciences Inc.) para determinação do $\mathrm{pH}$ e análises dos teores de $\mathrm{Ca}$ e Mg (espectrometria de absorção atômica) e de K (fotometria de chama). Os resultados de cada

Quadro 1. Composição dos substratos sintéticos preparados com diferentes proporções de areia com caulinita natural ou goethita sintética

\begin{tabular}{|c|c|c|c|c|c|c|}
\hline Tratamento & Areia & Caulinita & Goethita & Superfície específica(1) & Área adicionada(2) & pH água(3) \\
\hline & 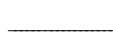 & $-\mathrm{g} \mathrm{kg}^{-1}$ & - & $\mathrm{m}^{2} \mathrm{~g}^{-1}$ & $\mathrm{~m}^{2} \mathrm{~kg}^{-1}$ & \\
\hline Areia & 1.000 & - & - & 0,71 & - & 7,2 \\
\hline Areia $+0,1 \%$ goethita & 999 & - & 01 & 0,86 & 153,2 & 7,2 \\
\hline Areia $+0,5 \%$ goethita & 995 & - & 05 & 1,48 & 765,9 & 7,5 \\
\hline Areia + 1, $0 \%$ goethita & 990 & - & 10 & 2,24 & 1531,9 & 7,6 \\
\hline Areia $+0,1 \%$ caulinita & 999 & 01 & - & 0,74 & 30,2 & 7,1 \\
\hline Areia $+1,0 \%$ caulinita & 990 & 10 & - & 1,01 & 302,3 & 7,2 \\
\hline Areia $+5,0 \%$ caulinita & 950 & 50 & - & 2,22 & 1509,5 & 7,2 \\
\hline
\end{tabular}

\footnotetext{
(1) Superfície específica dos substratos, considerando a proporção da mistura (areia $=0,71 \mathrm{~m}^{2} \mathrm{~g}^{-1}$; caulinita $=30,9 \mathrm{~m}^{2} \mathrm{~g}^{-1}$ e goethita $=$ $\left.153,9 \mathrm{~m}^{2} \mathrm{~g}^{-1}\right)$. (2) Área superficial adicionada nos substratos em relação à área do tratamento somente com areia. ${ }^{(3)} \mathrm{pH}_{\mathrm{H}_{2} \mathrm{O}}{ }$ dos substratos (relação 1:2,5), após a adição de palha de milho $\left(5 \mathrm{~g} \mathrm{~kg}^{-1}\right)$.
} 
tratamento foram convertidos em massa de cada elemento liberado por massa de substrato, sendo as médias acumuladas no tempo, juntamente com o respectivo erro-padrão da média.

O delineamento estatístico para os tratamentos foi um fatorial hierárquico completamente casualizado, considerando como fatores o componente inorgânico (caulinita ou goethita) e a proporção no substrato $(0,1,1,0$ e 5,0\%, para caulinita, e 0,1, 0,5 e $1,0 \%$, para a goethita). Após a análise da variância $(P<0,05)$, as médias da razão entre as massas liberadas de carbono, $\mathrm{Ca}^{2+}, \mathrm{Mg}^{2+} \mathrm{e} \mathrm{K}^{+}$e a massa de substrato, dentro da época considerada, foram relacionadas com a massa do componente inorgânico presente em cada tubo, incluindo o tratamento somente com areia, ajustando-se uma regressão linear simples.

Após duzentos dias de incubação, o material foi retirado dos tubos, seco em estufa a $50^{\circ} \mathrm{C}$ e nele determinado o teor de carbono orgânico total remanescente nas misturas pelo método WalkleyBlack, descrito em Tedesco et al. (1995). Para avaliar a influência do tipo de componente no acúmulo de carbono, a massa de carbono orgânico total retida no substrato ao final do experimento também foi relacionada com a massa de cada componente inorgânico e com a área superficial adicionada em cada tratamento.

\section{RESULTADOSE DISCUSSÃO}

$\mathrm{Na}$ figura 1, encontram-se as razões entre as massas acumuladas de $\mathrm{Ca}^{2+}, \mathrm{Mg}^{2+} \mathrm{eK}^{+}$percoladas durante 113 dias de decomposição da palha e a massa de substrato com diferentes proporções de caulinita ou goethita, de acordo com o tempo de decomposição. Para o $\mathrm{Ca}^{2+}, \mathrm{Mg}^{2+}$, houve efeito da caulinita e da goethita durante todo o período de avaliação $(P<0,01)$, tendo massas crescentes de cada componente proporcionado a liberação de menor massa dos elementos. Para oK ${ }^{+}$, não houve efeito de diferentes massas de goethita na retenção do elemento ( $P=0,62)$, mas, sim, com a presença de massas crescentes da caulinita $(P<0,01)$. Nos tratamentos em que as doses da caulinita e de goethita eram idênticas $(0,1$ e $1,0 \%)$, a magnitude da retenção dos el ementos liberados pela palha foi diferente para cada componente inorgânico.

A retenção de $\mathrm{Ca}^{2+}, \mathrm{Mg}^{2+}$ e $\mathrm{K}^{+}$, avaliada pelas massas liberadas ao final do período de avaliação, relacionou-se diretamente com a massa da caulinita ou da goethita em cada tratamento (Figura 2), e, portanto, foi dependenteda superfície para adsorção, exceto para o $\mathrm{K}^{+}$na goethita (Figuras 1 e 2).

Como o pH da solução percolada foi bastante similar entre tratamentos ao longo do período de avaliação (entre 7 e 8), podem-se utilizar os coeficientes angulares das equações indicadas na figura 2, que representam a massa retida pela caulinita ou goethita por unidade de massa adicionada nos tubos, para comparar o efeito dos componentes inorgânicos. A goethita reteve aproximadamente 22,6 vezes mais $\mathrm{Ca}$ e 11 vezes mais Mg do que a caulinita. Somente parte dessas diferenças pode ser explicada pela maior superfície específica da goethita em relação à da caulinita (153,9 contra 30,9 $\left.\mathrm{m}^{2} \mathrm{~g}^{-1}\right)$, pois essa relação é de

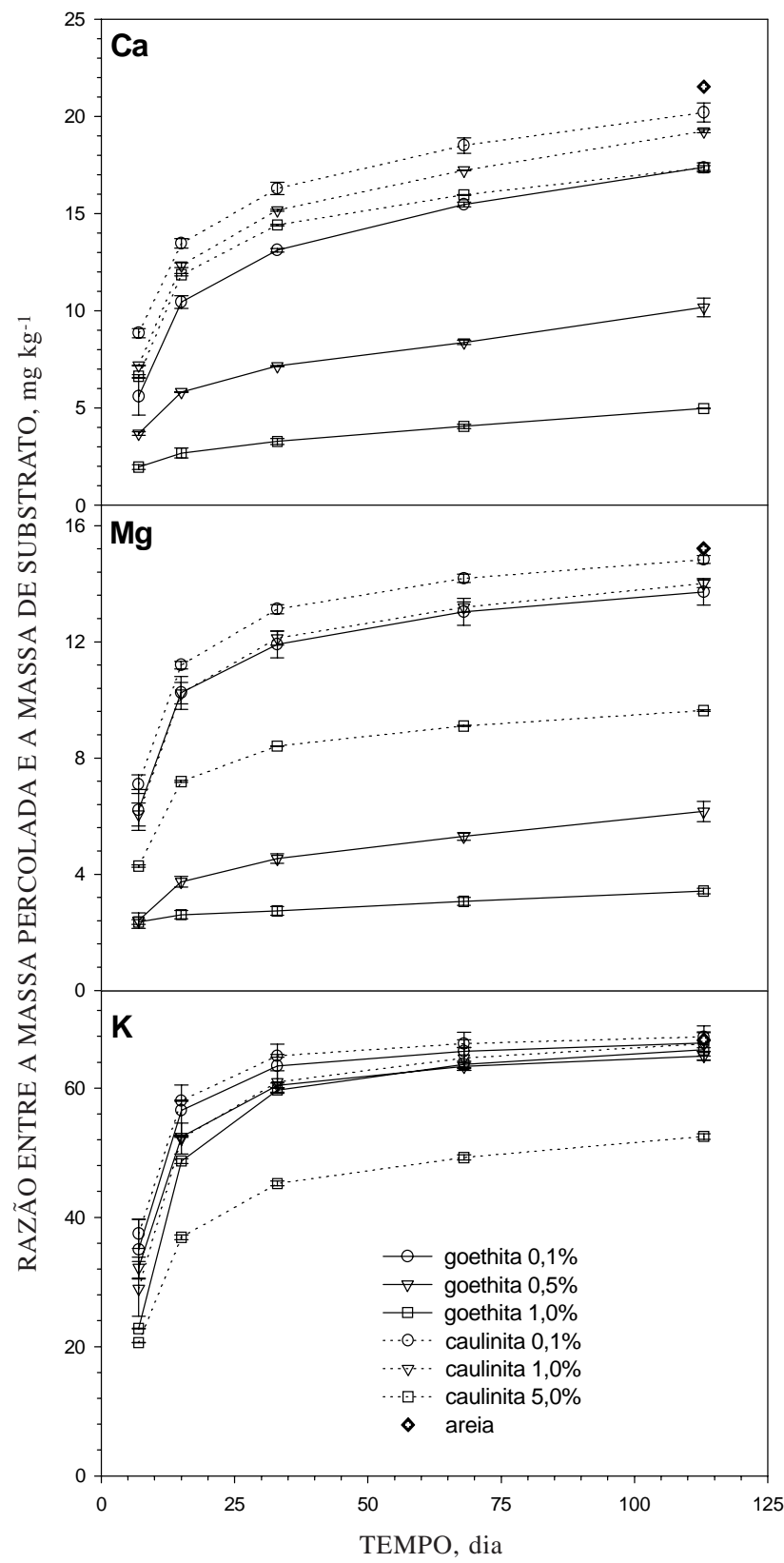

Figura 1. Razão entreas massas percoladas acumuladas de $\mathrm{Ca}$, Mg e $\mathrm{K}$ e a massa de substrato com diferentes proporções de goethita sintética e caulinita natural, considerando o tempo de decomposição de palha de milho (as barras verticais representam o erro-padrão da média). 
aproximadamente cinco vezes. O K+ teve comportamento inverso, sendo retido preferencialmente pela caulinita em relação à goethita (Figura 2).

Aliado a diferente superfície específica, o comportamento entre os componentes inorgânicos pode estar relacionado com os diferentes tipos de grupos funcionais nos seus planos de superfície (Stumm, 1992), resultando em diferenças na capacidade de adsorção e afinidade pelos el ementos.

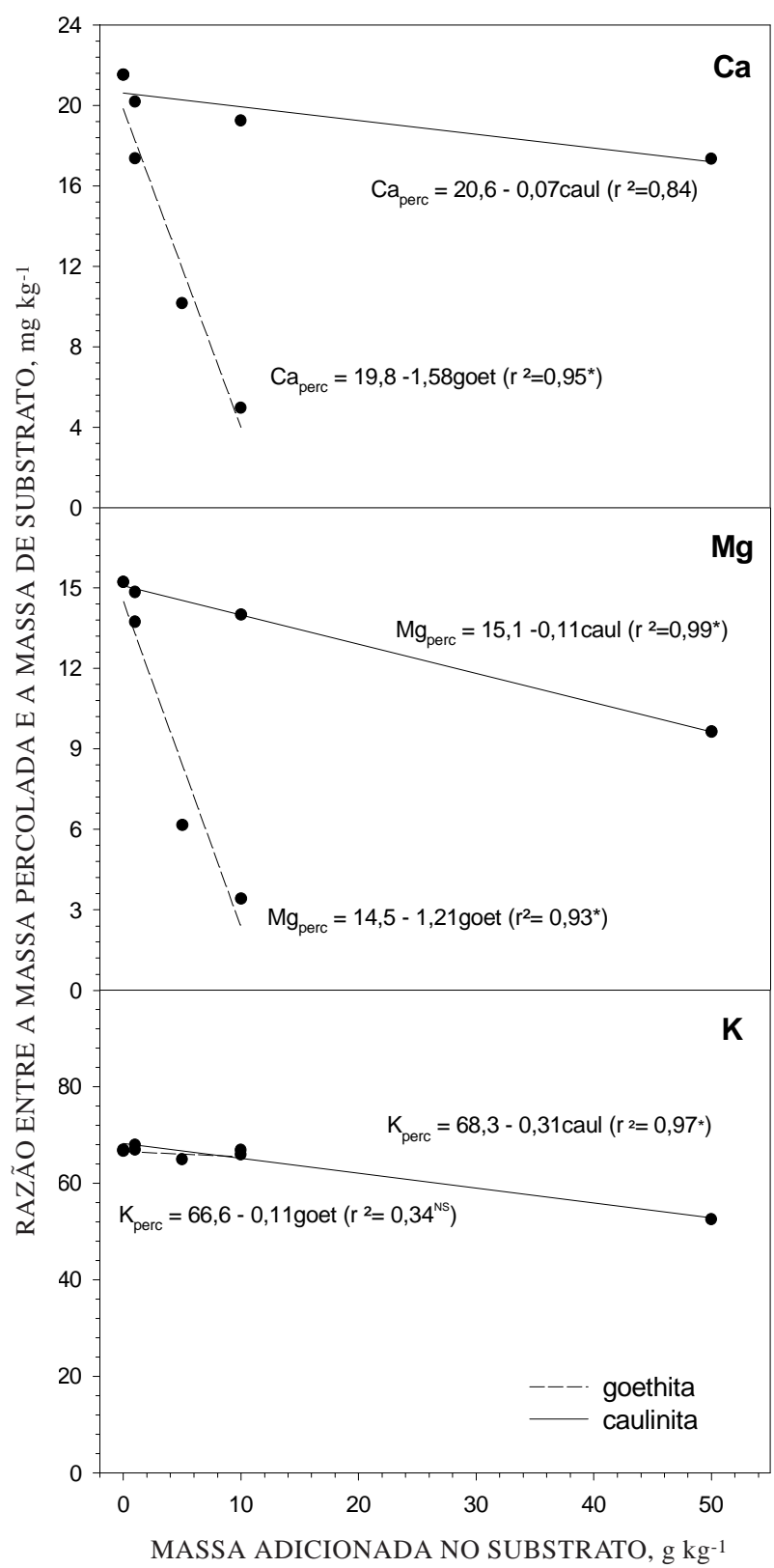

Figura 2. Razão entre as massas percoladas (perc) acumuladas de $\mathrm{Ca}, \mathrm{Mg}$ e $\mathrm{K}$, após 113 dias de decomposi ção de palha de mil ho, consi derando a massa adicionada de goethita sintética (goet) ou caulinita natural (caul) em substratos inorgânicos (* $P<0,05$ e ${ }^{\text {NS }}$ não-significativo).
A goethita apresenta carga superficial dependente do $\mathrm{pH}$, gerada por um único tipo de grupo funcional de superfície (Fe-OH da periferia do mineral), e a retenção dos cátions concordou com a ordem de afinidade dos íons esperada para superfícies de óxidos de ferro (Sposito, 1989), considerando o tamanho do raio não hidratado dos íons (Stumm, 1992). A caulinita, que reteve mais $K^{+} e m$ relação à goethita, pode apresentar cargas permanentes (substituição isomórfica do Si por Al na lâmina tetraedral exposta) e pH-dependente (grupos Al-OH eSi-OH das bordas eAl-OH da superfície octaedral exposta) (Stumm, 1992).

Dessa forma, apesar de o pH semelhante e a adsorção desses el ementos ocorrerem pela formação de complexos de esfera externa com a superfície dos minerais (Sposito, 1989), os componentes inorgânicos estudados apresentam diferentes grupos funcionais, com características distintas, principal menteácidobase (Stumm, 1992), que podem determinar diferente densidade de carga e afinidade pelos elementos nos sítios de troca.

Deve-se considerar ainda ser comum atribuir parte das cargas de superfície das caul initas naturais à contaminação com pequenas quantidades de filossilicatos 2:1 (Wieland \& Stumm, 1992), que podem adsorver especificamente íons de $\mathrm{K}^{+}$nas posições de intercamadas desses minerais, dependendo da baixa energia de hidratação do el emento em relação ao $\mathrm{Ca}^{2+} \mathrm{eMg}^{2+}$ (Douglas, 1989).

Outra consideração importante, além da diferença na superfície dos minerais, é que houve liberação de diferentes quantidades de cada el emento pela palha, em virtude de sua composição e do curso da decomposição nos tubos ao longo do tempo, o que pode influenciar a retenção dos cátions pelo efeito de massa.

Da mesma forma como verificado para $\mathrm{Ca}^{2+}, \mathrm{Mg}^{2+}$ eK+, as proporções de caulinita ou goethita também influenciaram a massa de carbonoliberada ao final do período de decomposição da palha (dados não apresentados, $\mathrm{P}<0,01$ ). Entretanto, pel ofato de esse elemento encontrar-se na solução do solo em diferentes formas, as quais podem ser bastante susceptíveis à atividade biológica, utilizaram-se somente os valores das massas acumuladas nos primeiros quinze dias (duas percolações), para avaliar a sua retenção pela goethita ou caulinita, período no qual a atividade biológica deve ter exercido menor interferência na interação do carbono com a superfície.

A retenção do carbono foi diretamente relacionada com a massa de cada componente inorgânico (Figura 3a), mas com magnitude diferente entre eles. Ao se comparar a massa de carbono percolada com a massa adicionada, a caulinita precisou de uma quantidade, aproximadamente, cinco vezes maior para proporcionar a mesma retenção obti da para goethita. Essa relação ésimi lar 
à encontrada para a superfície específica da caulinita em relação à da goethita (Quadro 1). Quando essa comparação é feita com a área adicionada nos tubos, cal culada segundo a proporção de cada componente em cada tratamento (Figura $3 b$ ), verifica-se que as massas de carbono retidas mostram-se dependentes da superfície específica dos componentes inorgânicos utilizados.

O principal mecanismo de adsorção de carbono orgânico solúvel pelos diferentes tipos de óxidos éa troca de ligantes, com o deslocamento do $\mathrm{OH}^{-}$do grupo funcional de superfície e a coordenação do metal da estrutura mineral com o oxigênio dos grupos carboxílicos ou hidroxílicos do composto orgânico (J ardine et al., 1989; Murphy et al., 1990; Gu et al., 1994). Esse ti po de ligação não requer que o composto apresente carga (Schulthess \& Huang, 1991). J á para a caulinita, o mecanismo de adsorção depende do tipo de carga e do grupo funcional a ser

MASSA ADICIONADA NO SUBSTRATO, $\mathrm{g} \mathrm{kg}^{-1}$

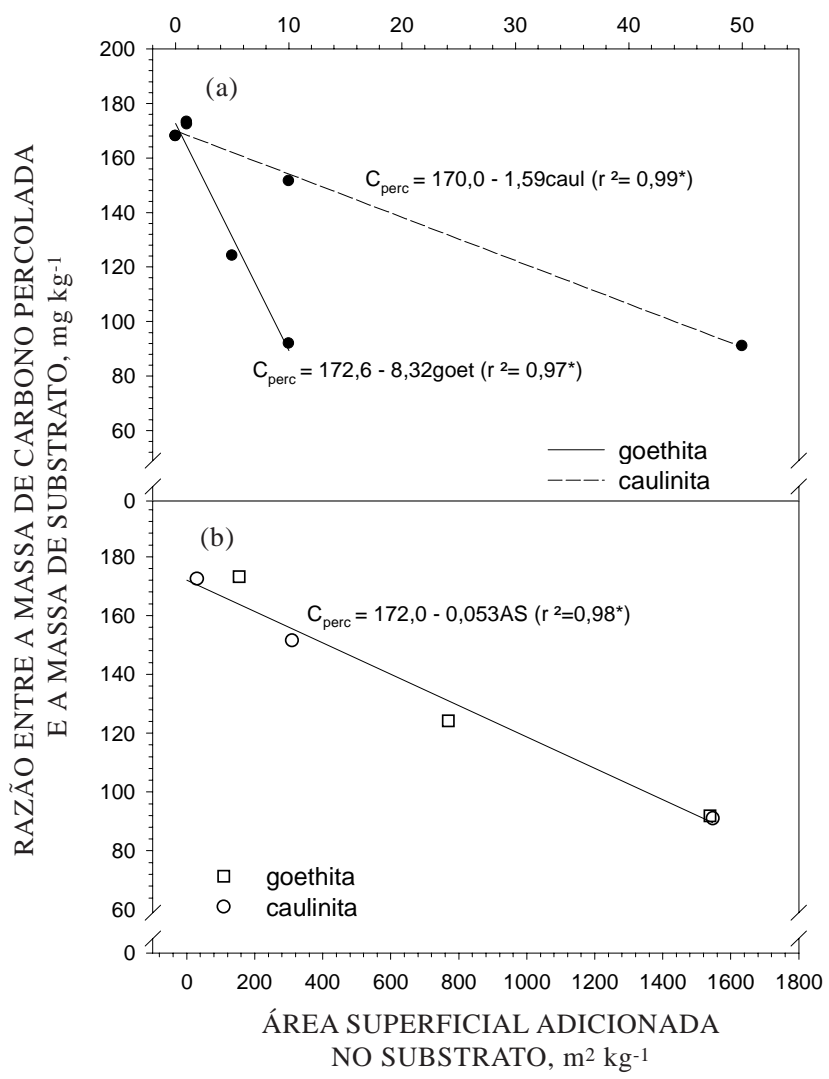

Figura 3. Razão entrea massa percolada acumulada de carbono orgânico solúvel $\left(C_{\text {perc }}\right)$ nas duas primeiras percolações ( 7 e 15 dias de decomposição de palha de milho), considerando a massa de caulinita natural (caul) ou goethita sintética (goet) (a) e área superficial (AS) (b) adicionada nos substratos inorgânicos (* $P<0,05$ e NS não-significativo). considerado. Nas superfícies com carga negativa permanente, o principal mecanismo seria a formação de pontes com cátions pol ivalentes (principal mente $\mathrm{Ca}^{2+} \mathrm{e} \mathrm{Mg}^{2+}$, em sol os próximos à neutral idade, eAl nos sol os ácidos) entre o mineral egrupos funcionais desprotonados das moléculas orgânicas (Oades, 1988).

A adsorção nas cargas $\mathrm{pH}$-dependentes das bordas deveria ter um comportamento semelhante ao seu óxido correspondente (Schulthess \& Huang, 1991). Entretanto, Varadachari et al. (1991), estudando o efeito de diferentes cátions trocáveis na adsorção de substâncias húmicas, revelaram ser a ligação direta de ácido húmico com a caulinita muito pequena ou ausente. Segundo os autores, houve incremento na adsor ção pel o mineral somente na presença de Ca e Al. Essa hipótese foi mantida no estudo de Varadachari et al. (1995) no qual determinaram que a participação das bordas dos cristais da caulinita na complexação húmusargilomineral ocorre por meio de ponte de cátions.

Apesar de a literatura relatar a ocorrência de diferentes mecanismos preferenciais de adsorção de carbono para diferentes tipos de superfíciemineral, os resultados obtidos neste estudo indicaram que somente a superfície específica disponível já é um fator importante para determinar a capacidade de adsorção de carbono em diferentes superfícies. I sso pode estar relacionado com a grande diversidade de compostos orgânicos que contêm carbono que são liberados pelos resíduos vegetais durante a decomposição e com a possibilidade de ocorrer interação dos minerais por diferentes mecanismos.

Embora o valor encontrado, em torno de $0,05 \mathrm{mg} \mathrm{m}^{-2}$ de $\mathrm{C}$ (coeficiente angular da figura $3 \mathrm{~b}$ ), esteja próximo do encontrado por Gu et al. (1994), para retenção de matéria orgânica em óxido de Fe com pH da solução próximo a 8, e isto indicar determinada capacidade de retenção de carbono orgânico solúvel pelos materiais, esses autores afirmaram que essa capacidade é extremamente influenciada pelo $\mathrm{pH}$ do meio e pela presença de outros ligantes aniônicos, podendo ser até oito vezes maior $\left(0,40 \mathrm{mg} \mathrm{m}^{-2}\right.$ de $\mathrm{C}$ em pH 3,0 na ausência de fosfatos e sulfatos).

Segundo J ardine et al. (1989) e Gu et al. (1994), essa imobilização do carbono orgânico solúvel por meio de compl exos com a superfície de minerais teria grande influência na mobilidade de eventuais contaminantes orgânicos ou inorgânicos a ele associados durante o transporte no perfil do solo.

O carbono orgânico total que permaneceu no material contido nos tubos, ao final do período de incubação, também foi dependente das proporções de goethita e de caulinita nos substratos (F igura $4 a$ ) e da área superficial adicionada pelo material acondicionado nos tubos (Figura 4b). O acúmulo de carbono orgânicototal pode ser explicado, em parte, pela retenção do carbono solúvel (Figura 3). 
De acordo com Gu et al. (1994), o carbono adsorvido por grupos funcionais de óxidos é dificilmente dessorvido, considerando o valor do $\mathrm{pH}$ e da força iônica. Dessa forma, a adsorção por minerais deve proporcionar maior proteção contra a decomposição pel os microrganismos e, juntamente com outros fatores, diminuir a taxa de decomposição dos resíduos orgânicos adicionados.

De maneira geral, a textura de um solo pode afetar a decomposição de um material orgânico por alterar a disponibilidade de água, distribuição do tamanho de poros, disponibilidade de nutrientes e área superficial, alterando a atividade biológica (Scott et al. 1996). Essa é uma das razões para que o modelo CENTURY, apresentado por Parton et al. (1987), considere a proporção de silte mais argila como um dos fatores que controla a taxa de estabilização do carbono no compartimento recalcitrante da matéria orgânica.

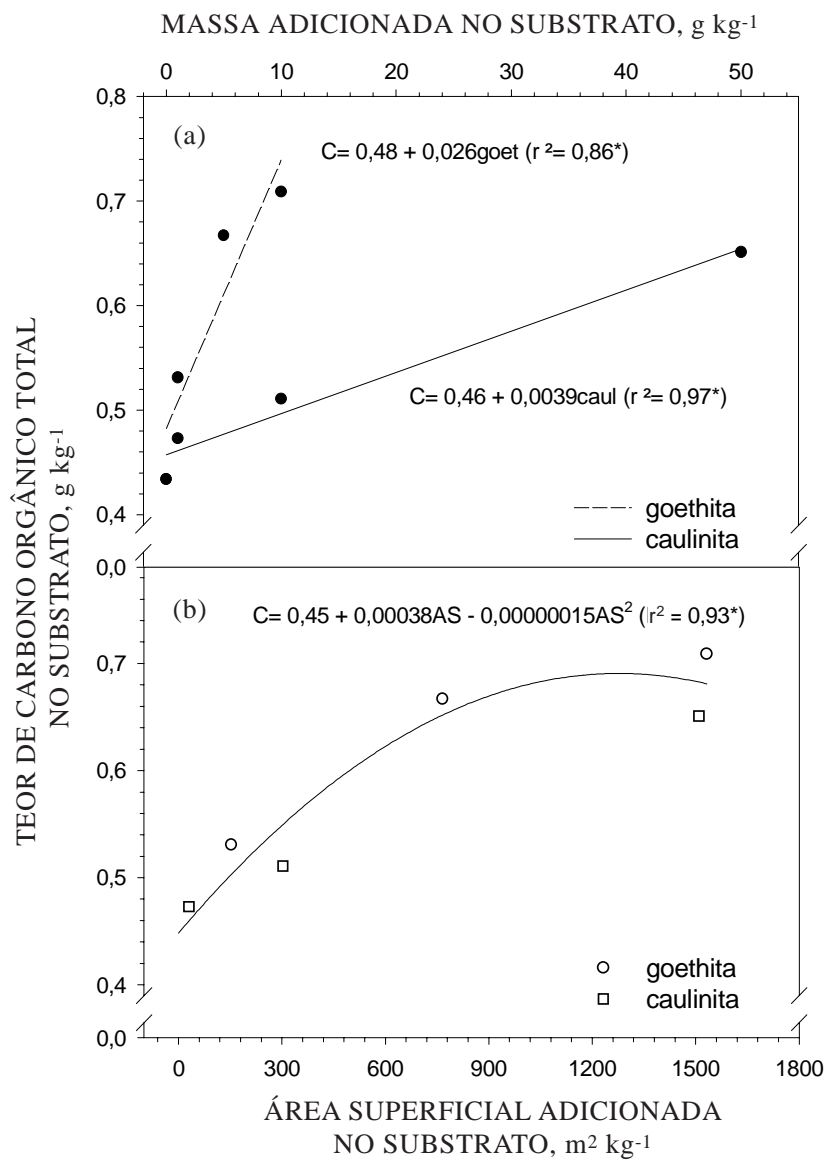

Figura 4. Teor de carbono orgânico total nos substratos, após $\mathbf{2 0 0}$ dias da adição de palha de milho, considerando a massa de caulinita natural (caul) ou goethita sintética (goet) (a) e da área superficial (AS) (b) adicionada nos substratos inorgânicos (* $P<0,05$ e ${ }^{\text {NS }}$ nãosignificativo).
Dessa forma, os resultados deste trabalho evidenciam que o tipo de componente inorgânico presente na fração argila de um solo constitui fator importante a ser considerado em modelos de predição da dinâmica da matéria orgânica do solo.

Os resultados obtidos neste estudo indicam que o preparo de substratos que simulem a composição do solo pode ser uma estratégia valiosa para isolar efeitos de componentes do solo. Por outro lado, os resultados devem ser avaliados com cuidado, tendo em vista a relativa artificialidade dos componentes e as condições experimentais utilizadas em relação ao ambiente do solo.

\section{CONCLUSÕES}

1. A retenção de $\mathrm{Ca}^{2+}, \mathrm{Mg}^{2+}, \mathrm{K}^{+}$e carbono, liberados durantea decomposição da pal ha de milho, foi diretamente relacionada com a proporção de caulinita natural egoethita sintética nos substratos inorgânicos. No caso dos cátions, a intensidade de retenção foi diferente entre os minerais utilizados, independentemente da superfície específica.

2. A goethita foi mais efetiva do que a caulinita para reter carbono da solução do sol o e acumular carbono orgânico total, sendo sua maior reatividade diretamente relacionada com sua superfície específica.

\section{LITE RATURA CITADA}

CORNEJ O, J. \& HERMOSÍN, M.C. Interaction of humic substances and soil clays. In: PICCOLO, A., ed. Humic substances in terrestrial ecosystems. Amsterdam, Elsevier, 1996. p.595-624.

DOUGLAS, L.A. Vermiculites. In: DIXON, J.B. \& WEED, S.B., eds. Mineral in soil environments. 2.ed. Wisconsin, Soil Science Society of America, 1989. p.635-674.

GREENLAND, D.J . I nteractions between humic and fulvic acids and clays. Soil Sci., 111:34-41, 1971.

GU, B.; SCHMITT, J .; CHEN, Z.; LIANG, L. \& MCCARTHY, J .F. Adsorption and desorption of natural organic matter on iron oxide: mechanisms and models. Environ. Sci. Technol., 28:38-46, 1994.

J ARDINE, P.M.; WEBER, N.L. \& MCCARTHY, J .F. Mechanisms of dissolved organic carbon adsorption on soil. Soil Sci. Soc. AmJ ., 53:1378-1385, 1989.

MARTIN, J .P. \& HAIDER, K. Influence of mineral colloids on turnover rates of soil organic carbon. In: HUANG, P.M. \& SCHNITZER, M., eds. Interactions of soil minerals with natural organics and microbes. Madison, Soil Science Society of America, 1986. p.283-304.

MOORE, T.R. The spectrophotometric determination of dissolved organic carbon in peat waters. Soil Sci. Soc. Am. J ., 49:15901592, 1985. 
MORTLAND, M.M. Mechanisms of adsorption of non humic organic species by clays. I n: HUANG, P.M. \& SCHNITZER, $M .$, eds. Interactions of soil minerals with natural organics and microbes. Madison, Soil Science Society of America, 1986. p.59-76.

MURPHY, E.M.; ZACHARA, J .M. \& SMITH, S.C. Influence of mineral-bound humic substances on the sorption of hidrophobic organic compounds. Environ. Sci. Technol., 24:1507-1516, 1990.

OADES, J.M. The retention of organic matter in soils. Biogeochem., 5:35-70, 1988.

OADES, J .M.; GILMAM, G.P. \& UEHARA, G. Interactions of soil organic matter and variable-charge clays. In: COLEMAN, D.C.; OADES, J .M. \& UEHARA, G., eds. Dynamics of soil organic matter in tropical ecosystems. Honolulu, University of Hawaii Press, 1989. p.69-95.

PARTON, W.J .; SCHIMEL, D.S.; COLE, C.V. \& OJ IMA, D.S. Analysis of factors controlling soil organic matter levels in Great Plains Grasslands. Soil Sci. Soc. Am. J ., 51:1173-1179, 1987.

QUIRK, J .P. Significance of surface areas calculated from water vapour sorption isotherms by the use of B.E.T. equation. Soil Sci., 80:423-430, 1955.

SCHULTHESS, C.P. \& HUANG, C.P. Humic and fulvic acid adsorption by silicon and aluminum oxide surfaces on clay minerals. Soil Sci. Soc. Am. J ., 55:34-42, 1991.

SCOTT, N.A.; COLE ,C.V.; ELLIOT, E.T. \& HUFFMAN, S.A. Soil textural control on decomposition and soil organic matter dynamics. Soil Sci. Soc. Am. J ., 60:1102-1109, 1996.
SPOSITO, G. The chemistry of soils. New York, Oxford Univ. Press., 1989. 277p.

STEVENSON, F.J. Humus chemistry: genesis, composition, reactions. 2.ed. New York, J ohn Wiley \& Sons, 1994. 496p.

STUM M, W. Chemistry of the solid-water interface. New York, J ohn Wiley \& Sons, 1992. 428p.

TEDESCO, M.J .; GIANELLO, C.; BISSANI, C.A.; BOHNEN, H. \& VOLKWEISS, S.J. Análises de solo, planta e outros materiais. 2.ed. Porto Alegre, Universidade Federal do Rio Grande do Sul, 1995. 174p. (Boletim técnico, 5)

VARADACHARI, C.; MONDAL, A.H. \& GHOSH, K. Someaspects of clay-humus complexation: effect of exchangeable cations and lattice charge. Soil Sci., 151:220-227, 1991.

VARADACHARI, C.; MONDAL, A.H. \& GHOSH, K. Theinfluence of crystal edges on clay-humus complexation. Soil Sci., 159:185-190, 1995.

VASUDEVAN, D. \& STONE, A.T. Adsorption of dissolved organic ligands. In: HUANG, P.M., ed. Soil chemistry and ecosystem health. Madison, Soil Science Society of America, 1998. p.133-156.

WIELAND, E. \& STUMM, W. Dissolution kinetics of kaolinitein acidic aqueous solutions at $25^{\circ} \mathrm{C}$. Geochim. \& Cosmochim. Acta, 56:3339-3355, 1992.

ZECH, W.; SENESI, N.; GUGGENBERGER, G.; KAISER, K.; LEHMANN, J .; MIANO, T.M.; MILTNER, A. \& SCHROTH, G. Factors controlling humification and mineralization of soil organic matter in the tropics. Geoderma, 79:117-161, 1997. 